\title{
LA UNIVERSIDAD PÚBLICA EN LA SOCIEDAD DEL CONOCIMIENTO
}

\author{
PUBLIC UNIVERSITY IN SOCIETY KNOWLEDGE \\ Máximo Ugarte Vega Centeno* \\ Docente Principal de la Facultad de Administración \\ Universidad Nacional Mayor de San Marcos-UNMSM / Lima-Perú \\ [Recepción: Mayo de 2013/ Conformidad: Junio de 2013]
}

\section{RESUMEN}

El debate sobre la universidad sigue vigente, la situación actual es preocupante por muchos factores internos y externos que influyen en su gestión y sus perspectivas de desarrollo hacia el futuro en una sociedad cambiante, en la que la universidad debe estar a la altura de los acontecimientos para responder y cumplir el rol que tiene asignado en la sociedad.

Palabras claves: universidad y conocimientos

\begin{abstract}
The debate about the university is still in force, the current situation is worrying for many internal and external factors that influence its management and its development prospects for the future in a changing society, in which the university must keep up the events to respond and fulfill the role assigned to it in society.
\end{abstract}

Keywords: university and knowledge.

* Diplomado en Gestión por el Programa de Alta Dirección (PAD), Universidad Privada de Piura. Magíster por la Universidad de Barcelona (España) y Doctor en Estudios Internacionales por la Universidad de Córdoba (España). Profesor Principal de la Escuela de Negocios Internacionales de la Universidad Nacional Mayor de San Marcos. 


\section{INTRODUCCIÓN}

Cuando hablamos de globalización de la economía y la sociedad del conocimiento es preciso vincularlos con las universidades, porque la globalizaciones exigente y llegó a estos centros de estudios; por lo tanto, las universidades y escuelas de posgrado tienen y deben estar a la altura de las exigencias.

América Latina está comprometida en este complejo proceso de transformaciones económicas y sociales, en esta aldea global donde los conocimientos están al frente de estos cambios y el sistema universitario tiene que modificar sus organizaciones para responder a los nuevos desafíos.

El sistema universitario en el Perú no está al margen de estos acontecimientos y para insertarse en este escenario competitivo es necesario estar preparados y programar estrategias que nos ayuden a superar estos desafíos, como la capacitación de los recursos humanos, la competitividad en base a la calidad de la enseñanza, la investigación y la actualización de los planes de estudios, acceso al mercado laboral de los egresados y las tecnologías de información y comunicación como socias en este proceso de inserción.

Es ese contexto debemos asumir como uno de los retos que la articulación de ciencia, tecnología y conocimiento son los elementos claves para el funcionamiento de un sistema universitario. Cualquier reflexión o acción relacionada con ellos, en cuanto a su contenido, cantidad, calidad, oportunidad, actualidad, pertinencia, manera de gestionarlo, transmitirlo, adquirirlo, entre otros, desempeñará un papel esencial en el mejoramiento de la calidad de la sociedad a la cual se debe.

\section{HIPÓTESIS}

La situación actual y los problemas de la universidad y los posgrados se deben a la influencia de factores externos e internos que influyen en su gestión y en sus perspectivas de desarrollo.

\section{ANÁLISIS Y DISCUSIÓN}

Las particularidades del estudio nos llevan a tener en consideración las siguientes variables de la hipótesis planteada:

1. La situación actual de la universidad y los problemas que viene enfrentando.

2. Los principales factores de su entorno que influyen en la gestión de la universidad.

3. Cuáles serían las perspectivas que tiene o debe tener la universidad y las escuelas de posgrado.

\section{La situación actual de la universidad y los pro- blemas enfrenta}

Podemos sintetizar su situación y problemas en los siguientes aspectos:

a) Estructura universitaria. Antes de 1930 existían nueve universidades públicas y una privada; hoy tenemos en el Perú más de 129 universidades de diverso tipo; 51 son nacionales (públicas) y 78 son privadas, además de una universidad municipal ${ }^{1}$.

b) Población. En 1960 se matricularon 30,000 alumnos aproximadamente, y en la actualidad, según la ANR, contamos con más de 700,000 estudiantes y cada año ingresan al sistema universitario un promedio de 90,000 estudiantes. Según el último censo universitario, el número de estudiantes de pregrado de las 35 universidades públicas se aproxima a los 8000,000 , distribuidos en más de 200 carreras profesionales ${ }^{2}$, y en el posgrado, son alrededor de 25,000 estudiantes.

c) Presupuesto. Cómo vamos a enfrentar el déficit presupuestal de las universidades para que cumplan los objetivos para los que fueron creadas es uno de los problemas principales, ya que el gobierno brinda escaso apoyo para incrementar la inversión en educación, y la mayoría de las universidades subvencionan los estudios ya que los pagos por derecho de matrícula son casi simbólicos. Las universidades públicas que recibieron mayor dinero según el presupuesto aprobado para el 2012 son la Universidad Nacional Mayor de San Marcos (S/. 360,598,804), la Uni-

1 Véase Informe 2011: Dirección de estadística y Planificación universitaria de la Asamblea Nacional de Rectores

2 Asamblea Nacional de Rectores .Informe de 07 de marzo del 2012. 
versidad Nacional de San Antonio Abad del Cusco $(S / .159,550,602)$ y la Universidad Nacional de Ingeniería (S/. 219,532,038). En el otro extremo del presupuesto se encuentran la Universidad Nacional de la Frontera, la Autónoma de Chota y la Intercultural de la Selva Central Juan Santos Atahualpa, las que reciben $S / .3,500,000$, que es el mismo monto que recibieron en el presupuesto del 2011 (ver Cuadro N. ${ }^{\circ}$ ). En cuanto a las fuentes de financiamiento de las universidades públicas, más o menos el $58 \%$ de su presupuesto asignado proviene de la recaudación tributaria del Estado. El 30\% corresponde a recursos directamente recaudados por las mismas universidades, es decir tasas, ventas de bienes, prestaciones de servicios, entre otros; $9 \%$ responde a donaciones y transferencias, y $3 \%$ a canon ${ }^{3}$ y sobre canon, regalías, rentas de aduanas y participaciones. Es de remarcar que no todas las universidades reciben canon minero, solo aquella ubicada en los departamentos donde existe actividad minera. A las universidades públicas se les debe dar mayores recursos y a la vez exigirles a que inviertan en ciencia y tecnología, así como a las universidades privadas con fines de lucro es necesario exigirles que paguen sus impuestos y que no se archive el proyecto que derogaba la Ley N. ${ }^{29766}$, que extendió los beneficios tributarios a las instituciones educativas, beneficio que consistía en un crédito tributario por reinversión del $30 \%$, lo que impidió al Estado cobrar S/.200 millones en el 2012. d) Democracia. La actual democracia elitista y excluyente no es representativa ni es suficiente. Las elecciones de las autoridades, como el Rector, Vicerrectores, Decanos de Facultades y de Escuela de Posgrado, así como el propio Comité Electoral, deberían ser por voto universal, aunque ello no resuelva el problema de la universidad. e) Calidad. Al hablar de calidad nos referimos a la condición que tiene todo servicio público o privado de la educación y, en particular, a la manera como se brinda ese servicio, en este caso la docencia, investigación y la proyección social. En el sistema universitario no existe un organismo especializado de acreditación para las universidades, con capacidad de decisión y seguimiento del conjunto de normas y procedimientos estructurados que puedan definir $y$ establecer los criterios, estándares y procesos de evaluación, acreditación y certificación, con la finalidad de asegurar los niveles básicos de calidad que debe brindar una institución universitaria.

f) Investigación. La inversión en investigación no solo es insuficiente sino casi nula. El Perú invierte el $031 \%$ de nuestro PBI en este rubro, mientras que Brasil invierte 1.1\%, Estados Unidos, el 2.7\%, y Finlandia, el 3.5\%.

g) Normatividad jurídica. Las universidades en el Perú pueden ser públicas o privadas y se basan en la actual Ley Universitaria N. ${ }^{\circ} 23733$ de 1986, que regula las universidades. En 1996 se promulga el Decreto Legislativo N. ${ }^{\circ} 882$, mediante el cual se aprueba la Ley de Promoción de la Inversión en la Educación. La Ley señala que un claustro puede crearse luego de un estudio de mercado y de factibilidad de por medio; sin embargo, el Decreto Legislativo N. ${ }^{\circ} 882$ amplía las posibilidades cuando señala que las universidades privadas podrían ser constituidas a iniciativas de su promotor y las públicas mediante una ley, a condición de que su creación sea sustentada y que sea aprobada por el CONAFU, al que le corresponde vigilar por su cumplimiento, ya que esta institución evalúa a las privadas y hace seguimiento a las públicas. Hoy en día ambas normas resultan desfasadas porque no corresponden a los requerimientos y proyección de la universidad.

3 La ONG Ciudadanos al Día (CAD) informa que al presupuesto aprobado para el período 2012 se añadirán US\$ 336 millones por concepto ce canon, tributo correspondiente al $20 \%$ del total de ingresos y rentas obtenidos por el Estado por la explotación de yacimientos mineros, petroleros y gasíferos. Estos fondos van a actividades de investigación y desarrollo de ciencia y tecnología en las universidades públicas de las zonas de influencia de dichos recursos naturales. Ver, Informe. CAD, enero del 2012.

4 Desde otro ámbito, en el Perú las leyes de educación no ayudan a que la formación técnica alcance un prestigio en el mercado laboral. En ese sentido el prof. Thahtemberg indica que no se permite convalidar ni reconocer los estudios de carrera técnica en un instituto como equivalentes a los semestres que se cursan en una universidad, por lo tanto no pueden tener un título equivalente al universitario y entonces no pueden hacer luego un posgrado, ver en La educación dual es una modalidad de enseñanza que se realiza en dos lugares: El centro educativo superior y la empresa, véase Empresas- Negocios. Educación para el trabajo cada vez pierde competitividad; en Revista de la cámara de Comercio de Lima N. ${ }^{\circ} 540$, Lima 2012, p. 6 y 7. 
h) Mercado laboral. Según un estudio de la Organización Internacional del trabajo (OIT), alrededor de 1 300,000 jóvenes en el Perú no estudian ni trabajan $^{4}$, asimismo tomando en cuenta la constante y vertiginosa transformación actual del mercado de trabajo, hay que considerar, como cierto, la rapidez con la que los conocimientos se vuelven obsoletas ${ }^{5}$. La universidad, en general, no responde a las exigencias y necesidades de las empresas públicas y privadas en el país.

g) Número de egresados. Los egresados no corresponden al número de graduados con tesis en pregrado y posgrado. Ante los bajos índices de investigación, se propone que el estudiante ya no opte por el examen de grado sino por una tesis productiva, aunque el artículo 23 de la actual Ley Universitaria da la posibilidad de obtener el título de licenciado o sus equivalentes mediante la presentación de una tesis o un examen profesional.

i) Manejo de idiomas. La exigencia científica no solo demanda el manejo de idiomas sino también la demanda laboral como parte de la globalización de la ciencia y las universidades, así como en el ejercicio profesional, cultural y hasta deportivo; por lo tanto, el idioma no deja de ser una referencia en la inserción en el mercado laboral nacional e internacional que cada día es más exigente. Ya no es sostenible tener una carrera con el manejo intermedio de un idioma.

j) El rol de los intelectuales y la universidad. En su formación académica la universidad debe brindar instrumentos de conceptualización y teorización que estén al alcance de los participantes, que les permita pensar, reflexionar y analizar objetivamente y con libertad. Una universidad no cumpliría su rol si descuidara la búsqueda libre del conocimiento y su aplicación a la realidad social de su país, región o localidad. k) Credibilidad. Si tenemos que recuperar la credibilidad de las universidades, es necesario que triunfe la meritocracia y la idoneidad; para esto sería importante empezar con transparencia ${ }^{6}$ el ingreso y nombramiento, ascensos y ratificaciones de catedráticos. Por último, y lo que más nos preocupa son tres aspectos de mucha trascendencia en la universidad, nos estamos refiriendo a la gestión, liderazgo y cultura organizacional que viene a ser el talón de Aquiles de las universidades en general.

\section{Factores del entorno que influyen en la uni- versidad}

Todos somos conscientes de los distintos factores que influyen en la universidad, tanto a nivel interno como externo. Como factores internos podemos citar, entre otros, las diferencias académicas, falta de docentes idóneos, deserción estudiantil, falta de diálogo y tolerancia, resistencia al cambio, etc.

En lo externo; por un lado, tenemos influencias como el gobierno, las empresas públicas y privadas, la sociedad, los profesores, estudiantes, trabajadores, y el entorno familiar. Y, por otro lado, está la globalización de las universidades en la sociedad del conocimiento, el ingreso de las universidades extranjeras al mercado nacional ofreciendo posgrados en forma tradicional y a distancia. Necesitamos impulsar una revolución en la educación y la modernización del capital humano, que empiece a cambiar la mentalidad de los profesores y alumnos, involucrando en ese proceso a la familia y a la sociedad en general.

Las universidades y los posgrados tienen que afrontar la competitividad con una formación de profesionales que puedan que puedan responder a las exigencias actuales; en otras palabras, tienen que avanzar de una visión regional a una internacional.

5 Beneitone, Pablo y otros (2007). Op. Cit. p. 24. Ver World Bank Group. (2004). "Key Development Data \& Statistics 2004”. Citado en la revista: Conduct in an Emerging Market Economy. Documento en línea: http://www.ita.doc.gov/goodgovernance/adobe/Bus_Ethics_sp/ Chapter\%20I/I_Chapter_1.pdf. Consulta el 19 de noviembre del 2011. Vease,, UDUAL Universidades. (2004). Documento en línea: http:// redalyc.uaemex.mx/redalyc/pdf/373/37302702.pdf. México, enero a junio.

6 Ver la Ley N.2 27806, Ley de Transparencia y Acceso a la Información. Esta ley viene a ser un documento que regula las instituciones públicas sobre la información pertinente y de carácter público de las actividades y servicios que brinda.

7 Véase, Castillo Maza, Juan. Gestión de la Educación Virtual. En: Gestión en el Tercer Milenio, Revista de Investigación de la Facultad de Ciencias Administrativas de la Universidad Nacional Mayor de San Marcos. No 19. Lima, Facultad de Ciencias Administrativas de la UNMSM, Julio del 2007, pág. 85-92.

78/ QVIPUKAMAVOC | Vol. 21(39) 2013 
La tecnología de la información y comunicaciones (NTC) han incorporado nuevos retos y posibilidades en el ámbito de la educación ${ }^{7}$ como soporte para potenciar la interrelación entre los miembros de la comunidad académica (correo electrónico, Internet, educación a distancia, bibliotecas en línea, aulas virtuales, etc.) es fundamental junto a la capacitación constante para que no sigan proliferando los nuevos semianalfabetos modernos en el manejo de la tecnología y los idiomas.

También influyen las nuevas exigencias como la competitividad y no la competencia (ser mejor que el otro); es decir, la capacidad de gestión humana basada en la competitividad, donde las organizaciones como las universidades tienen retos para definir su visión, misión, estructura, estrategias y recursos, acciones orientadas a lograr mayores niveles de competitividad. Para el logro de estos propósitos se requiere de una estrategia humana orientada a ser generadora de valores a través de sus diferentes procesos como la selección, capacitación y desarrollo, compensación, gestión de desempeño, diseño de cargos, reclutamiento, entre otros; asimismo, las perspectivas de aprender algo nuevo, junto a la psicología de la capacitación para el éxito, nos permitirán romper las barreras psicológicas de carácter decisivo para todas la formas de cambio. Todas esta redefiniciones exigirán terminar con el modelo tradicional.

Entonces surge la pregunta ¿cómo medir la competitividad de las universidades y de las escuelas de posgrado? Simplemente se realizará una medición cuantitativa (calidad y acreditación educativa igual a competitividad de las universidades) y cualitativa (los valores que son inherentes a cada institución). Otras formas de medir la competitividad podrían ser la formación (responsabilidad de sus profesionales en la sociedad), investigación (publicaciones), proyección (actividades de extensión) y gestión (organización de su producción de bienes y servicios).

\section{Perspectivas de la universidad y las escuelas de posgrado}

Al concluir las diferentes reformas en la década de los noventa en el ámbito de la educación superior en el país se caracterizó por la creciente educación superior privada y la masificación de la matrícula, alto índice de egresados, y el creciente equilibrio de géneros de estudiantes y profesionales, así como la universidad mediante su cogobierno y autonomía ${ }^{8}$ no han podido todavía integrarse con la sociedad y tampoco desligarse de su total dependencia del presupuesto público y la disputa con el sector privado de la educación por captar estudiantes para la obtención de recursos propios que en su mayoría es para financiar los gastos salariales en la Universidad. En ese contexto las universidades se han visto en la imperiosa necesidad de buscar nuevas alternativas como la Autoevaluación y la Acreditación de las Universidades para superar los problemas que han surgido y fundamentalmente en relación a la calidad en la educación que son el origen del bajo nivel de calidad de los profesionales.

En esa orientación, podemos decir, en primer lugar, antes las universidades podían evaluar y acreditar su calidad mediante diferentes criterios y sistemas de acreditación, como el norteamericano y el de la Asamblea Nacional de Rectores ${ }^{9}$, a través del Instituto de Investigación y Fomento de la Modernización y Acreditación Universitaria (ANR-INFOMACU, 2001). Hoy la acreditación de la calidad educativa se basa en la Ley N. ${ }^{\circ} 28740$, Ley del Sistema de Acreditación y Certificación de Calidad Educativa (SINEACE) y su respectivo reglamento ${ }^{10}$. Existen otros tipos de acreditación en el ámbito académico, como:

8 Véanse al respecto Ramírez Prado Fidel. La autonomía universitaria; Ortiz Caballero, René. El sentido y Alcance de la autonomía Universitaria. Rodríguez Chávez, Iván. La Autonomía universitaria en la legislación universitaria vigente. Boccio de la Paz, Benjamin. Factores e la Autonomía universitaria. En: Hacia una nueva universidad en el Perú (2003). García zarate, Oscar -Compilador -, Lima, Fondo editorial de la UNMSM. También del mismo autor véase en: porunamejoruniversidad blogpot.com. Pdf., Junio de 2010.

9 Véase, Asamblea Nacional e Rectores. Comisión Nacional de rectores para la acreditación, Dirección general de Investigaciones y acreditación Universitaria. Dcto. De trabajo. Estándares Mínimos para la Autoevaluación de las carreras profesionales; Edit.ANR, Lima, Perú, 204

10 Ley 28740, promulgada el 19 de Mayo de 2006 y publicado su Reglamento el 10 de Julio de 2007. 


\subsection{Acreditación especializada por programa.}

Mediante este mecanismo se acreditan generalmente carreras que tengan relación e impacto con la salud, la seguridad y el bienestar social.

3.2. Acreditación Institucional. Por el que se acreditan las características. En general, se evalúan los recursos, servicios, el proyecto educativo, la capacidad de gestión y la situación financiera, entre otros. Se dice que mayormente es complementaria y posterior a la acreditación por programas.

3.3. Acreditación de profesionales. Permite autenticar la competencia técnica de los titulados de un programa para el ejercicio profesional, responsabilidad casi siempre asumida por las asociaciones profesionales quienes establecen los mecanismos para evaluar.

Bien, entonces antes de definir la autoevaluación, podemos decir que la acreditación se consigue como resultado del desarrollo de tres momentos básicos: la autoevaluación, la evaluación por pares externos la acreditación.

La Universidad Nacional Mayor de San Marcos define la autoevaluación como una etapa del proceso de acreditación en la que la propia institución se evalúa internamente, según normas o estándares de calidad que tienden a ser de alcance internacional, con el objeto de determinar su cumplimiento o incumplimiento, especificando las áreas en las que se requiere mejorar. Esta actividad la desarrollan comités nombrados por la institución o unidad académica, los cuales realizan su tarea mediante guías previamente definidas, coordinando la recopilación de la información, su análisis y la elaboración del informe de autoevaluación, todo esto junto a la motivación interna, el respaldo institucional y el liderazgo y participación plena de todos los miembros de la
Universidad ${ }^{11}$, valores sin los cuales no sería exitoso el proceso de autoevaluación ${ }^{12}$.

Esto es, este proceso se convierte en un componente fundamental para poder describir, emitir o validar juicios de valor sobre la institución, o nos ayuda a examinar planes, acciones y logros, comprobar e interpretar los logros de un programa y/o mejorar un programa o curso de acción para que responda a las necesidades de un mercado laboral, en la búsqueda del desarrollo económico y social del país.

En términos generales, todo proceso de evaluación de un programa de acreditación ${ }^{13}$ presupone, entre otros, definir los propósitos de la evaluación; delimitar sus variables, elementos, indicadores y criterios, para establecer el nivel y calidad del programa de funcionamiento, así como recolectar, analizar e interpretar información pertinente; valorar la calidad del programa en su totalidad o en algunas de sus variables y elementos; analizar el logro del programa evaluado; identificar e incorporar los ajustes y cambios pertinentes; $y$ establecer los procesos permanentes de seguimiento y control.

En el caso de las escuelas de posgrado, según opiniones de instituciones y otros expertos, se pueden medir por diferentes modalidades como la autoevaluación interna y externa. En el primer caso, la organización, ejecución y supervisión del programa de autoevaluación tiene que estar bajo responsabilidad de personas que tengan el interés y compromiso con el programa. En el segundo, participarán especialistas ajenos al programa, identificando los problemas, las dificultades y sugiriendo más no haciendo correcciones al programa ${ }^{14}$. Si como proceso global la autoevaluación sirve para medir las fortalezas y debilidades de una institución; entonces, para llegar a ese objetivo, tenemos que apoyarnos en un conjunto de factores

11 Ver Universidad Nacional Mayor de San Marcos. (2006). Lineamientos para una Política de Calidad, Autoevaluación y Acreditación en la UNMSM. Documento de Trabajo, p.73.

12 Véase, Gutiérrez Huby, Ana María. (2006). “Formulación y desarrollo de un modelo de autoevaluación para la acreditación del Programa de Contabilidad”. Tesis para optar el Grado Académico de Magíster en Administración, mención Gestión Empresarial. Lima, UNMSM, p. 32.

$13 \mathrm{Al}$ respecto véase también, Cabrejos Polo, Jorge; Gutiérrez Huby, Ana maría y López Más, Julio. (2006). Guía de Autoevaluación para la acreditación en el área economico empresarial. Lima, Editorial UNMSM. Ver, Oficina Central de Calidad Académica y Acreditación de la UNMSM (2005). Hacia la Autoevaluación en San Marcos. Lima, Editorial UNMSM.

14 Véase Consorcio de Universidades (1999). Autoevaluación institucional: Manual para Instituciones de educación superior. Lima, Editorial Fondo Editorial Universidad del Pacífico, pp. 58 y ss. 
propuestos en un modelo de matriz de autoevaluación, que luego pueden ser agrupados en criterios e indicadores de gestión de calidad, entonces surge la interrogante: ¿qué son y para qué sirve los indicadores en la autoevaluación?

Podemos decir que un indicador viene a ser un elemento de medición que permite aclarar y medir en el tiempo un concepto; la expresión indicador también se refiere a los datos cuantitativos y cualitativos (números, medidas, opiniones, hechos, percepciones) que nos ayudarán a tener en cuenta lo que pueda estar sucediendo en el ámbito o sector que nos interesa conocer. Asimismo, decimos que sirve porque nos permitirá medir los cambios o situaciones a través del tiempo. Estos instrumentos son importantes también porque, a través de ellos, podemos evaluar y dar inicio a los procesos de desarrollo para alcanzar y orientar mejores resultados en la autoevaluación.

En cambio, la acreditación es el reconocimiento formal y público, por parte de un organismo oficial autorizado, de la competencia técnica de una entidad para la realización de una actividad perfectamente definida. Se trata, pues, de un proceso a través del cual se busca comprobar que la institución por acreditar desarrolla sus actividades en función de su plan estratégico, además de certificar periódicamente el cumplimiento de un conjunto de estándares mínimos de calidad previamente establecidos. Consecuentemente, la acreditación propicia el mejoramiento de la calidad, idoneidad, pertinencia y solidez de la institución, e incentiva en sus integrantes una cultura de evaluación permanente ${ }^{15}$.

Entre los objetivos que busca la acreditación, principalmente, está el ser un mecanismo para que las instituciones de educación superior rindan cuentas a la sociedad y el Estado, dar fe pública de la calidad de las instituciones y de los programas de educación superior; asimismo, sobre el servicio educativo que prestan, brindar información confiable a los usuarios y ser un instrumento para que las instituciones verifiquen el cumplimiento de su misión, sus propósitos, objetivos, en el marco de la Constitución y la Ley, y de acuerdo a sus estatutos.

En ese sentido implica certificar públicamente los requerimientos mínimos de calidad que reúne un programa o una institución académica sin la cual no podría mejorar el sistema universitario del país; en otras palabras, la acreditación tiene como propósito garantizar la calidad de las instituciones o programas de la universidad y ayudarlos a superar sus niveles de calidad con el mejoramiento continuo, entendiendo el mejoramiento como una filosofía que ha rebasado al ámbito empresarial y que se aplica también en el sector educativo, por lo mismo que todos pretendemos progresar hacia el autoexamen mediante mejoras graduales y constantes que brotan como iniciativas de las actividades diarias y que son parte de las acciones frecuentes en el ámbito laboral. $\mathrm{Al}$ margen de las definiciones, lo que nos interesa es que se desarrolle una cultura de mejoramiento continuo (elemento indispensable para el mejoramiento) en todos los estamentos (profesores, estudiantes y administrativos) de la universidad, desterrando la soberbia organizacional, el miedo al cambio, la falta de tolerancia y la poca participación del personal ${ }^{16}$.

En esa orientación se pueden medir indicadores y criterios para establecer el nivel de calidad del programa en vigencia; la gestión académica y administrativa de la universidad y sus posgrados; grados académicos de sus profesores; selección y admisión de alumnos; las distintas responsabilidades que asumen los graduados en las instituciones públicas y privadas de la sociedad; el currículo; el impacto social; la investigación; número de publicaciones especializadas; horas de atención a los alumnos; infraestructura de sus aulas, bibliotecas, laboratorios, cafeterías, instalaciones deportivas; sistema de apoyo para la búsqueda de apoyo, entre otros. En resumen, se busca medir la

15 Ver Universidad Nacional Mayor de San Marcos. (2006). Lineamientos para una Política de Calidad, Autoevaluación y Acreditación en la UNMSM. Documento de trabajo, p.76.

16 Sobre acreditación también se puede ver: De Paz Toledo, Zenón y Cuba Sancho, Juana. (2003). Lineamientos para una política de calidad, autoevaluación y acreditación. Lima, Editorial UNMSM. Véase, Consorcio de Universidades. (1999). Autoevaluación institucional: Manual para Instituciones de educación superior. Lima, Editorial Fondo Editorial Universidad del Pacífico. 
eficiencia en la organización académica y de gestión de la universidad y el posgrado.

Ahora bien, para poder acreditar la calidad educativa ${ }^{17}$ se tienen que plantear objetivos dentro de un plan estratégico como formar profesionales de altísima calidad; la internacionalización con soportes de alianzas estratégicas con otras universidades; mejorar el posicionamiento en cuanto a los competidores; desarrollar programas de investigación con participación de docentes y estudiantes y/o en convenios de cooperación nacional e internacional; modernizar las currículas de las facultades y los syllabus de los cursos con un adecuado apoyo; modernizar la gestión con diferentes acciones a tomar en función de las políticas sobre administración, eficiencia y productividad; ética profesional, elemento fundamental para generar conciencia de sus responsabilidades en su desarrollo social, la producción y el fortalecimiento de una cultura organizacional; formar líderes con una visión compartida y una cultura institucional de acuerdo a la exigencia de los cambios en la escena de investigación, la rigurosidad, la tecnología y el desarrollo de competencias; incorporar en su gestión estratégica universitaria la necesidad de vincular a la universidad con la sociedad, no solo a nivel de sus representantes, sino de toda la comunidad universitaria, generando acciones institucionales que respondan a las necesidades de toda la sociedad.

Podemos decir, entonces, que las universidades y sus escuelas de posgrado deben ser dinámicas, creativas e innovadoras, para que junto a la tecnología de las comunicaciones y todas las herramientas virtuales puedan ser canalizadas a través del blogs, Twitter, Facebook, Google plus, Linkedin y otras redes sociales, que permitan acercar la calidad de investigación, de enseñanza y de gestión. En esa orientación diríamos que no cerremos las puertas del cambio porque estaríamos negando toda posibilidad de progreso.

Antes de concluir, quiero señalar que las lecciones aprendidas del pasado son importantes, como dice el refrán: "uno aprende y después desaprende para aprender solo"; en ese sentido, les invitamos a tener una visión compartida hacia un proyecto nacional de universidad y de escuelas de posgrado; a actuar y comprometer nuestras voluntades para superar la situación actual de la universidad y para afrontar la competitividad, así como el fomento y exigencia de la modernización a través de la autoevaluación y acreditación con el propósito de garantizar la calidad de las universidades y programas del posgrado en el Perú.

Podemos afirmar una vez más, que la universidad cumple un rol fundamental en la sociedad del conocimiento y no podemos estar de espaldas a la realidad; todavía quedan muchas interrogantes en el tintero, como por ejemplo: ¿cuál es el compromiso social de la universidad frente a los nuevos enfoques de desarrollo científico, tecnológico y de gestión del conocimiento, en un contexto de globalización de las economías de mercado?, ¿seguiremos pensando sin dar nuevos lineamientos sobre el nuevo contexto social, económico y político en el que se encuentra la universidad peruana?, ¿a qué distancia se encuentra la universidad latinoamericana frente a los avances en ciencia, tecnología y gestión de conocimientos en los países desarrollados y emergentes?, ¿cuáles son las estrategias impulsoras del cambio de paradigmas en la concepción y desarrollo de ciencia, tecnología y producción de conocimiento, que deberían ser asumidas por las universidades de la región? Y si el cristal con que se mire fuera ciencia-tecnología-conocimiento; entonces, ¿cómo nos vemos a través de él como universidad peruana frente a los escenarios universitarios de otros países?

Responder a estas interrogantes nos llevará por caminos tal vez transitados pero no asimilados a plenitud, dado que las políticas de desarrollo en ciencia y tecnología no están articuladas al quehacer universitario y empresarial, tal como lo exigen las actuales tendencias del desarrollo social que privilegian el capital del conocimiento. Entendiendo que ciencia

17 Yarzábal, Luis (2002). “La Educación Superior en la sociedad contemporánea”. Conferencia dictada en el Primer Foro Nacional de la Educación Superior. Managua, mayo del 2002, En: Universidad y Sociedad, revista del Consejo Nacional de Universidades, N. ${ }^{\circ} 10$, septiembre del 2002. 
y tecnología son herramientas indispensables en la construcción de sociedades modernas e incluyentes, se hace necesaria fortalecer la investigación científica yla innovación tecnológica como tareas imprescindibles para apoyar el desarrollo de las sociedades y para competir en un entorno cada vez más dominado por el conocimiento y la información; escenario en el cual la universidad asume una alta responsabilidad social.

Para finalizar, queremos manifestar que al escribir lo hacemos sin triunfalismo ni pesimismos, simplemente describimos la realidad y a la vez seguimos pensando que la universidad es más grande que sus problemas. Sirvan pues, estas reflexiones para reiniciar el debate sobre las universidades y escuelas de posgrado, ya que desde la recuperación de la democracia, se ha esperado que algunas universidades se fortalezcan y otras reconstruyan su potencial en términos de docencia, investigación, publicaciones, y la vinculación con la sociedad a través de actividades de extensión y transferencia de tecnología, aunque los problemas siguen siendo económicos y de rentas, entre otros.

\section{CONCLUSIONES}

1. El concepto de universidad tradicional ha cambiado en esta sociedad del conocimiento y la globalización.

2. La universidad tienen como base la formación, investigación y responsabilidad social, así como la educación integral, la formación de valores y como resultado debe ser la calidad académica

3. La falta de apoyo presupuestal y de políticas erradas hacia el sistema universitario por parte del Estado están sumergiendo a la universidad en una crisis coyuntural y estructural. Es necesaria la realización de una reforma integral de la educación superior, que asegure la calidad de la formación académica, donde la investigación es tarea prioritaria, donde la investigación es tarea prioritaria y la universidad sea un centro de creación de conocimiento de manera permanente y sostenida.
4. Se crearon universidades públicas por réditos políticos, sin presupuesto propio y sin evaluar la calidad de la educación, sin criterio educacional ni de desarrollo. La mayoría de las nuevas universidades creadas, para seguir existiendo, necesitan que otras instituciones les transfieran parte de su infraestructura, ya que sus bienes están inscritos en el inventario de bienes de la universidad anterior, judicializándose muchas veces su puesta en marcha, generándose disputas de la sede y toma de locales; así van surgiendo mayores conflictos sociales. La proliferación de universidades va junto con la baja calidad educativa. Está demostrado que la tendencia a una masificación de la educación superior produce la pérdida de la calidad del aprendizaje, situación que se traduce en que los profesores tienden a adaptar la enseñanza al nivel de los alumnos promedio. A esto se suma que los profesores no solo tienen que hacer docencia sino que, además, investigación; pero aquí es necesario reflexionar en el nivel de aporte de las mismas a la mejora de la sociedad y a su vez, cómo llega el conocimiento a ella. La masificación y politización deteriora la calidad académica.

5. El desarrollo de la ciencia y la innovación debe ser promovida mediante la relación entre empresa privada, gobierno y universidades. En el ámbito de la investigación podríamos decir que el debate está en cómo desconcentrar los recursos de gestión y el uso de rentas para alcanzar una gestión de innovación, acortando la fisura digital para una mejor eficiencia.

6. La organización de las universidades requiere de cambio y desarrollo para una gestión administrativa transparente, eficiente y eficaz con participación democrática para una gestión que se base en una dirección estratégica con innovación y creatividad, en la búsqueda de una mejor formación profesional de excelencia y una concertación para el desarrollo. 
7. En la actualidad podemos afirmar que las universidades peruanas tienen dos retos fundamentales a enfrentar: la acreditación de la calidad educativa y el seguimiento homogéneo en su evaluación como una forma de generar una cultura de la calidad y del modelo educativo universitario.

8. Las universidades que no cumplan con los estándares internacionales y no estén acreditadas no podrán emitir títulos a nombre de la nación, solo certificados a nombre de su institución; de esta manera se exigirá y se incentivará el mejoramiento de la calidad educativa en la formación científica que cada universidad debe tener.

9. Se tiene que promulgar una nueva ley universitaria que obligue y financie la investigación en ciencia y tecnología y que, a su vez, desarrolle posgrados, ya que en la actualidad están diseñadas para ofrecer estudios de pre grado y son pocas las que dirigen sus recursos para desarrollar programas de especialización con nuevos conocimientos. La nueva Ley universitaria no solo es un imperativo legal sino ético.

10. En esta aldea global, la generación de ciencia y tecnología es un poder, para esto se requiere continuidad en la calidad en la formación y se necesita de herramientas como la predictibilidad, competencia y eficiencia de las universidades públicas. En ese sentido, podemos afirmar -en términos generales- que la educación y la innovación son el pasaporte para el futuro.

\section{REFERENCIAS BIBLIOGRÁFICAS}

1. Aguirre Yato, Víctor y otros (2007). Estrategias para el desarrollo de las Universidades del Perú. Lima, Asamblea Nacional de Rectores. Comisión Especial, designada por Resolución N. ${ }^{\circ} 368$ 2007-ANR.

2. Arboleda Toro, Néstor (2005). ABC de la Educación virtual y a distancia. E-Learning en la sociedad de la información, Bogotá-UNESCOIESALC.Asamblea Nacional de Rectores (ANR). (2011). Informe 2011: Dirección de Estadística y Planificación Universitaria de la Asamblea Nacional de Rectores. Lima.
3. Asamblea Nacional de Rectores. Comisión Nacional de Rectores para la Acreditación, Dirección general de Investigaciones y acreditación Universitaria (2004). Estándares Mínimos para la Autoevaluación de las carreras profesionales. Documento de Trabajo. Lima, Edit. ANR.

4. UNESCO (1995). Documento para el Cambio y el Desarrollo en la Educación Superior. París.

5. Bustamante Sánchez, Yolanda. La gestión del conocimiento y las organizaciones modernas. En: Gestión en el Tercer Milenio, Revista de Investigación de la Facultad de Ciencias Administrativas de la Universidad Nacional Mayor de San Marcos. N. ${ }^{\circ} 11$. Lima, Facultad de Ciencias Administrativas de la UNMSM, julio del 2003.

6. Burga Díaz, Manuel(2003). La actualidad de una larga historia: La responsabilidad ética y política de la Universidad; en: Hacia una nueva universidad en el Perú, García Zárate, Oscar (Compilador). Lima, Fondo Editorial de Universidad Nacional Mayor de San Marcos.

7. Brunner, J.J. (1998). Globalización Cultural y Postmodernidad, Breviarios. Santiago de Chile, Fondo de Cultura Económica.

8. Carnoy, Martín (1999). Globalization and educational reform: what planners need to know. París, IIP - UNESCO.

9. Carvallo, Constantino (2009). Diario Educar. Lima, Editorial Aguilar.

10. Castillo Maza, Juan. Gestión de la Educación Virtual. En: Gestión en el Tercer Milenio, Revista de Investigación de la Facultad de Ciencias Administrativas de la Universidad Nacional Mayor de San Marcos. No 19. Lima, Facultad de Ciencias Administrativas de la UNMSM, Julio del 2007.

11. Cisneros, Jaime Luis (2009). Aula Abierta. Lima, Editorial Norma; Penac.

12. Consorcio de Universidades. (1999). Autoevaluación institucional: Manual para Instituciones de educación superior. Lima, Fondo Editorial Universidad del Pacífico.

13. De Paz Toledo, Zenón y Cuba Sancho, Juana. (2003). Lineamientos Para una política de calidad, autoevaluación y acreditación. Lima, Editorial UNMSM. 
14. Delgado de la Flor Badaraco, Francisco y Alcázar C., Marcia. (2004). "Informe sobre Educación Superior Universitaria”. Lima, Asamblea Nacional de Rectores. Ver: Aguirre Yato, Víctor y otros. (2008). Estrategias para el desarrollo de las Universidades del Perú. Lima, Asamblea Nacional de Rectores.

15. Gutiérrez Huby, Ana María (2006). "Formulación y desarrollo de un modelo de autoevaluación para la acreditación del Programa de Contabilidad". Tesis para optar el Grado Académico de Magíster en Administración, mención Gestión Empresarial. Lima, UNMSM.

16. López Más, Julio; Cabrejos Polo, Jorge y Gutiérrez Huby, Ana María (2006). Guía de Autoevaluación para la acreditación en el área económico empresarial. Lima, Editorial UNMSM.

17. Ongaro Estrada, Andrés A. (2006). La Legislación sobre Educación Superior en el Perú. Lima, Edit. ANR.

18. Rama Vitale, Claudio (2009). Tendencias de la educación Superior en América Latina y el Caribe en el siglo XXI. Tomo II. Lima, Asamblea Nacional de Rectores.
19. Rama, Claudio (2004). La compleja iniquidad en la educación superior en América Latina y el Caribe. Caracas, Fondo Editorial IPASME.

\section{NORMAS LEGALES}

1. Constitución Política del Perú, de 7 de setiembre de 1993

2. Ley $\mathrm{N}^{\circ} 13417$, de 8 de abril de 1960 . Ley que estuvo vigente hasta el 18 de febrero de 1969

3. Ley N. ${ }^{\circ} 23733$, de 17 de diciembre de 1983 y vigente hasta la fecha y que no tuvo ningún interés sobre la acreditación universitaria.

4. Ley N. ${ }^{\circ} 23384$, General de Educación, de 18 de mayo de 1982.

5. Resoluciones de Asamblea Nacional de Rectores Nro.051-99-ANR de 4 de febrero de 1999.

6. Ley $\mathrm{N}^{\circ}$ 28740, promulgada el 19 de Mayo del 2006 y publicado su Reglamento el 10 de julio del 2007.

7. Ley N. ${ }^{\circ} 28044$, Ley General de Educación, de 16 de julio del 2003

8. Decreto Legislativo $\mathrm{N} .{ }^{\circ} 882$, Promoción de la Inversión de la educación, de 9 de noviembre de 1996.

9. Decreto Ley N. 17437 , de 18 de febrero de 1969. 